\title{
Author Correction: The hippocampus as a predictive map
}

Kimberly L Stachenfeld, Matthew M Botvinick (D) and Samuel J Gershman (D)

Correction to: Nature Neuroscience https://doi.org/10.1038/nn.4650, published online 02 October 2017.

In the version of this article initially published, equation (7) read

$$
M=\sum_{t=0}^{\infty} \gamma^{t} T^{t}=\left(I-\gamma T^{t}\right)^{-1}
$$

It should have read

$$
M=\sum_{t=0}^{\infty} \gamma^{t} T^{t}=(I-\gamma T)^{-1}
$$

The error has been corrected in the HTML and PDF versions of the article.

Published online: 25 April 2018

https://doi.org/10.1038/s41593-018-0133-1

\section{Publisher Correction: Medial preoptic circuit induces hunting-like actions to target objects and prey}

Sae-Geun Park, Yong-Cheol Jeong, Dae-Gun Kim, Min-Hyung Lee, Anna Shin, Geunhong Park, Jia Ryoo, Jiso Hong, Seohui Bae, Cheol-Hu Kim, Phill-Seung Lee (1) and Daesoo Kim (D)

Nature Neuroscience https://doi.org/10.1038/s41593-018-0072-x, published online 29 January 2018.

In the version of this article initially published, a sentence in the fifth paragraph of the Results read, "Immunohistochemistry revealed that VGLUT2 ${ }^{+}$MPA neurons rarely expressed CaMKII $\alpha$, which is a putative marker for subcortical glutamatergic neurons." It should have read, "Immunohistochemistry revealed that CaMKII $\alpha^{+}$MPA neurons rarely expressed VGLUT2, which is a putative marker for subcortical glutamatergic neurons." The error has been corrected in the HTML and PDF versions of the article. In the supplementary information originally posted online, the wrong version of Supplementary Fig. 1 was posted and some of the supplementary videos were interchanged. In the corrected Supplementary Fig. 1, the top right subpanel was added and the original Supplementary Fig. 1a was divided into 1a and $1 \mathrm{~b}$, with subsequent panels incremented accordingly. The legend was changed from "a. Schematic illustrating electrical lesioning of the rat anterior hypothalamus. Electrical lesion areas (gray) in five representative brain sections are depicted. Scale bar, $1 \mathrm{~mm}$ " to "a. Repetitive electrical stimulations of the anterior hypothalamus using bipolar electrodes (Left) caused a lesion at the hypothalamic area (middle, marked by asterisk) successfully in 7 rats (Right, overlapped images of brain sections located from the bregma $-0.24 \mathrm{~mm}$ ). Scale bar, $1 \mathrm{~mm}$. b. Electrical lesion areas (gray) in five representative brain sections from anterior to posterior are depicted." The errors have been corrected online.

Published online: 7 March 2018

https://doi.org/10.1038/s41593-018-0115-3

\section{Publisher Correction: An interactive framework for whole-brain maps at cellular resolution}

Daniel Fürth, Thomas Vaissière, Ourania Tzortzi, Yang Xuan, Antje Märtin, lakovos Lazaridis, Giada Spigolon, Gilberto Fisone, Raju Tomer, Karl Deisseroth, Marie Carlén, Courtney A. Miller, Gavin Rumbaugh and Konstantinos Meletis

Nature Neuroscience https://doi.org/10.1038/s41593-017-0027-7 (2017); published online 4 December 2017

In the version of this article initially published online, Daniel Fürth was not listed as a corresponding author. The error has been corrected in the print, PDF and HTML versions of this article. 